\title{
Identifying Olive Cultivars by Isozyme Analysis
}

\author{
Isabel Trujillo and Luis Rallo \\ Departamento de Agronomia, Universidad de Cordoba, Apartado 3048, Cordoba 14080, Spain
}

Pere Arús

Department de Genetica Vegetal, Institut de Recerca i Tecnologia Agroalimentaries, 08348 Cabrils, Barcelona, Spain

\begin{abstract}
Additional index words. Olea europaea, electrophoresis
Abstract. Pollen samples of 155 olive (Olea europaea L.) cultivars from different origins were analyzed to study isoenzymatic variability in five enzyme systems: alcohol dehydrogenase (ADH), esterase (EST), glucose phosphate isomerase (GPI), leucine aminopeptidase (LAP), and malic enzyme (ME) using starch gel electrophoresis. Polymorphism was observed in all of the isozyme systems. ME, GPI, EST, and LAP were the most useful systems for identification of cultivars. Different combinations of banding patterns of these systems allowed us to identify $85 \%$ of the cultivars. The remainder were separated into groups of two or three cultivars that could be identified using morphological characteristics. No intracultivar polymorphisms were observed.
\end{abstract}

The olive has been for millenia the most important oleaginous crop of the Mediterranean basin, where $95 \%$ of the cultivated world area occurs.

The outcrossing origin of the species and the introgression of genes from wild into cultivated olives (Zohary and Spiegel Roy, 1975) resulted in high diversity of cultivars in all mediterranean countries (Barranco and Rallo, 1984, 1985; Bottari and Spina, 1953; Leitão, 1988; Loussert and Brousse, 1980; Morettini, 1972; Valdeyron and Crossa-Raynaud, 1950). Systems of classification and identification based on quantitative and/or qualitative morphological characters were used by the above cited authors to study this diversity. Although these methods were effective, they presented practical drawbacks due to effect of environmental fluctuations on expression of most morphological traits. The use of biochemical markers such as isozymes overcome these problems since they are little affected by the environment and can easily be detected in a variety of tissues by relatively simple, rapid and inexpensive procedures. However, their use in cultivar identification requires the presence of high intercultivar isozyme polymorphism as well as the absence of intracultivar isozyme polymorphism (Weeden and Lamb, 1985).

Isozymes have been successfully used for cultivar identification in several fruit species as apricots (Byrne and Littleton, 1989), almond (Cerezo et al., 1989), cherimoya (Ellstrand and Lee, 1987; Pascual et al., 1993), mango (Degani and El Batsri, 1990), apple (Weeden and Lamb, 1985), and grape (Parfitt and Arulsekar, 1989; Stavrakakis and Loukas, 1983).

Pontikis et al. (1980) found high isoenzymatic variability in olive pollen samples of 27 cultivars, mostly of Greek origin. All cultivars could be identified with only two out of the sixteen enzyme systems used.

The objective of the present study was to identify a wide range of olive cultivars from 13 countries using isozyme analysis of pollen samples.

\section{Materials and Methods}

Pollen samples were collected from 155 cultivars growing at the germplasm collection of the Centro de Investigación y Desarrollo

Received for publication 19 May 1994. Accepted for publication 7 Sept. 1994. The cost of publishing this paper was defrayed in part by the payment of page charges. Under postal regulations, this paper therefore must be hereby marked advertisement solely to indicate this fact.
Agrario at Córdoba, Spain. Cultivar names, number of trees sampled, and origins are shown in Table 1.

Pollen was collected according to the method described by Pontikis et al. (1980) and stored at -20C until analyzed. Pollen samples $(40 \mathrm{mg})$ were crushed with $0.15 \mathrm{ml}$ extraction buffer $(0.61$ $\mathrm{M}$ tris HCL, $\mathrm{pH} 8$, containing $1 \% \mathrm{w} / \mathrm{v}$ reduced glutathione).

Electrophoretic analysis was performed on a horizontal slab (gel form: $23 \times 20 \times 1 \mathrm{~cm}, 12 \%$ Connaught hydrolyzed starch) according to a method described by Shields et al. (1983). The extracts were absorbed into paper wicks $(1.5 \times 0.3 \mathrm{~cm}$, Whatman no. 3) and inserted into a vertical cut in the gel.

Three gel-electrode buffers were used: tris-citrate, $\mathrm{pH} 7.8$ (TC 7.8); h-is-citrate, $\mathrm{pH} 7$ (TC 7); and histidine, $\mathrm{pH} 5.7$ (H 5.7). The composition of the buffers TC 7.8 and TC 7 are described in Shields et al. (1983). Histidine 5.7 gel buffer was prepared with $0.006 \mathrm{M}$, Lhistidine, $\mathrm{pH}$ 5.7. The electrode buffer for $\mathrm{H} 5.7$ gels was prepared with $0.13 \mathrm{M}$ tris and $0.04 \mathrm{M}$ citric acid, $\mathrm{pH} 7$. Electrophoresis was run under constant voltage at 4C. The voltage conditions were $100 \mathrm{~V}(\mathrm{H}$ 5.7) and $150 \mathrm{~V}$ (TC 7.8, TC 7) for the first $35 \mathrm{~min}$. At this point the paper wicks were then removed and the voltage was increased to 200 $\mathrm{V}$ (H 5.7) for $3 \mathrm{~h}$ and to $300 \mathrm{~V}$ (TC 7.8, TC 7) for $4 \mathrm{~h}$.

Optimal resolution was obtained when using TC 7.8 gels and corresponding electrode buffers for the staining of ADH (E.C. 1.1.1.1), EST (E.C. 3.1.1.-), LAP (E.C. 3.4.11.1), TC 7 for GPI (E.C. 5.3.1.9), and H 5.7 for ME (E.C. 1.1.1.40).

The gels were sliced horizontally after electrophoresis and immediately stained for enzyme activity according to the methods described for ADH and GPI by Vallejos ( 1983) and for EST and LAP by Mora and Serradilla ( 1985). ME gels were stained in $90 \mathrm{ml}$ $0.1 \mathrm{M}$ tris $\mathrm{pH} 8,10 \mathrm{mg}$ nicotinamide adenine dinucleotide phosphate (NADP), $20 \mathrm{mg}$ methyl thiazolyl tetrazolium (MTT), $5 \mathrm{mg}$ phenazine methosulfate (PMS), $10 \mathrm{ml} 1 \mathrm{M}$ sodium L-malate, $\mathrm{pH}$ 7.5 and $2 \mathrm{ml} 10 \% \mathrm{MgCl}_{2}$.

\section{Results and Discussion}

Polymorphism was observed in all enzyme systems for the cultivars studied. On the other hand, the banding patterns obtained in samples collected from the same trees in different years or from several trees of the same cultivars either from the same or different origin were consistent and showed no differences.

The zones of activity and bands detected were numbered according to their proximity to the anodal end. The intensity of the 
Table 1. Isozyme phenotypes of 155 olive cultivars.

\begin{tabular}{|c|c|c|c|c|c|c|c|c|}
\hline \multirow[b]{2}{*}{ Cultivar } & \multirow[b]{2}{*}{ Origin $^{y}$} & \multirow[b]{2}{*}{$\overline{\mathrm{ADH}}$} & \multirow[b]{2}{*}{ ME } & \multicolumn{3}{|c|}{ Banding patterns } & \multicolumn{2}{|c|}{ Identification ${ }^{x}$} \\
\hline & & & & EST & GPI & LAP & SO & $\mathrm{co}$ \\
\hline Aglandou & $\mathrm{Fr}$ & $\mathrm{A}$ & $\mathrm{S}$ & $\mathrm{B}$ & $\mathrm{E}$ & $\mathrm{A}-$ & $*$ & $*$ \\
\hline Alameño Blanco & $\mathrm{Sp}$ & A & $\mathrm{B}$ & $\mathrm{D}$ & $\mathrm{C}$ & $\mathrm{C}$ & $*$ & $*$ \\
\hline Alameño de Cabra & $\mathrm{Sp}$ & A & B & $\mathrm{F}$ & M & $\mathrm{H}$ & $*$ & $*$ \\
\hline Alameño de Marchena' & $\mathrm{Sp}$ & A & $\mathrm{D}$ & $\mathrm{D}$ & $\mathrm{C}$ & $\mathrm{D}$ & * & $*$ \\
\hline Alameño de Montilla (3) & $\mathrm{Sp}$ & $\mathrm{A}$ & $\mathrm{G}$ & $\mathrm{G}$ & $\mathrm{N}$ & $\mathrm{C}$ & $*$ & $*$ \\
\hline Amigdaloia & Gr' & A & $\mathrm{E}$ & $\mathrm{E}$ & $\mathrm{D}$ & $\mathrm{A}$ & $*$ & $*$ \\
\hline Arbequina (2) & $\mathrm{Sp}$ & A & $\mathrm{A}$ & M & $\mathrm{E}$ & $\mathrm{C}$ & $*$ & $*$ \\
\hline Ascolana Tenera & It & $\mathrm{A}$ & $\mathrm{A}$ & $\mathrm{G}$ & A & I & $*$ & $*$ \\
\hline Ayrouni & $\mathrm{Lb}$ & $\mathrm{A}$ & A & A & $\mathrm{C}$ & $\mathrm{C}$ & $\mathrm{f}$ & $*$ \\
\hline Azeitem & $\mathrm{Pt}$ & A & $\mathrm{E}$ & $\mathrm{D}$ & $\mathrm{D}$ & $\mathrm{C}$ & $*$ & $*$ \\
\hline Azul & $\mathrm{Sp}$ & A & I & $\mathrm{F}$ & $\mathrm{N}$ & $\mathrm{C}$ & $*$ & $*$ \\
\hline Beladi & $\mathrm{Lb}$ & $\mathrm{A}$ & $\mathrm{H}$ & B & G & $\mathrm{B}$ & $*$ & $*$ \\
\hline Blanqueta (2) & $\mathrm{Sp}$ & $\mathrm{A}$ & $\mathrm{J}$ & $\mathrm{F}$ & G & B & $*$ & $*$ \\
\hline Bouteillan & $\mathrm{Fr}$ & A & G & B & I & $\mathrm{A}$ & $*$ & $*$ \\
\hline Buidiego & $\mathrm{Sp}$ & $\mathrm{A}$ & Q & $\mathrm{R}$ & $\mathrm{C}$ & $\mathrm{D}$ & $*$ & $*$ \\
\hline Caballo & $\mathrm{Sp}$ & $\mathrm{A}$ & $\mathrm{T}$ & $\mathrm{A}$ & $\mathrm{D}$ & $\mathrm{C}$ & $*$ & $*$ \\
\hline Callosina & $\mathrm{Sp}$ & A & I & $\mathrm{C}$ & M & $\mathrm{H}$ & $*$ & $*$ \\
\hline Campanil & $\mathrm{Sp}$ & A & G & $\mathrm{F}$ & M & $\mathrm{D}$ & $*$ & $*$ \\
\hline Caninese & It & $\mathrm{B}$ & $\mathrm{J}$ & A & V & $\mathrm{A}$ & $*$ & $*$ \\
\hline Cañivano Blanco & $\mathrm{Sp}$ & A & B & $\mathrm{D}$ & $\mathrm{C}$ & $\mathrm{D}$ & a & * \\
\hline Cañivano Negro & $\mathrm{Sp}$ & $\mathrm{A}$ & A & $\mathrm{B}$ & $\mathrm{D}$ & $\mathrm{D}$ & $\mathrm{b}$ & $b$ \\
\hline Carboncella & It & A & $\mathrm{C}$ & $\mathrm{H}$ & $\mathrm{F}$ & $\mathrm{H}$ & $*$ & $*$ \\
\hline Carrasqueño de Alcaudete & $\mathrm{Sp}$ & A & A & $\mathrm{E}$ & $\mathrm{D}$ & $\mathrm{D}$ & * & $*$ \\
\hline Carrasqueño de Elvas & $\mathrm{Pt}$ & A & A & $\mathrm{D}$ & $\mathrm{C}$ & $\mathrm{D}$ & $*$ & $*$ \\
\hline Carrasqueño de la Sierra & $\mathrm{Sp}$ & A & $\mathrm{O}$ & $\mathrm{A}$ & $\mathrm{D}$ & $\mathrm{H}$ & $*$ & $*$ \\
\hline Carrasqueño de Lucena & $\mathrm{Sp}$ & A & A & $\mathrm{P}$ & $\mathrm{G}$ & $\mathrm{D}$ & $*$ & $*$ \\
\hline Cellina & It & A & $\mathrm{J}$ & $\mathrm{N}$ & Q & $\mathrm{F}$ & $*$ & $*$ \\
\hline Cerezuela & $\mathrm{Sp}$ & $\mathrm{A}$ & I & $\mathrm{B}$ & G & $\mathrm{D}$ & $* *$ & $*$ \\
\hline Cipresino & It & A & $\mathrm{K}$ & $\mathrm{D}$ & I & $\mathrm{G}$ & $*$ & $*$ \\
\hline Cobrançosa & $\mathrm{Pt}$ & $\mathrm{A}$ & $\mathrm{E}$ & $\mathrm{D}$ & $\mathbf{C}$ & $\mathrm{C}$ & $\mathrm{b}$ & $*$ \\
\hline Coratina & It & A & $\mathrm{J}$ & $\mathrm{T}$ & $\mathrm{J}$ & $\mathrm{G}$ & $*$ & $*$ \\
\hline Cordovil de Serpa & $\mathrm{Pt}$ & A & B & $\mathrm{E}$ & $\mathrm{D}$ & $\mathrm{D}$ & $\mathrm{k}$ & $*$ \\
\hline Cornezuelo (3) & $\mathrm{Sp}$ & A & $\mathrm{v}$ & $\mathrm{A}$ & $\mathrm{G}$ & $\mathrm{D}$ & $*$ & $*$ \\
\hline Cornicabra (4) & $\mathrm{Sp}$ & $\mathrm{A}$ & $\mathrm{G}$ & $\mathrm{F}$ & $\mathrm{G}$ & $\mathrm{D}$ & $*$ & $*$ \\
\hline Cornicabra Parda de Villena & $\mathrm{Sp}$ & A & $\mathrm{E}$ & $\mathrm{K}$ & $\mathrm{N}$ & $\mathrm{H}$ & $*$ & $*$ \\
\hline Chalkidiki & $\mathrm{Gr}$ & A & $\mathrm{V}$ & $\mathrm{B}$ & G & $\mathrm{H}$ & $*$ & $*$ \\
\hline Chami & $\mathrm{Sr}$ & A & $\mathrm{D}$ & $\mathrm{B}$ & $\mathrm{W}$ & A & $*$ & $*$ \\
\hline Changlot Real & $\mathrm{Sp}$ & A & $\mathrm{J}$ & B & $\mathrm{D}$ & $\mathrm{D}$ & c & c \\
\hline Chetoui & $\mathrm{Tn}$ & A & $\mathrm{C}$ & $\mathrm{s}$ & $\mathrm{J}$ & A & $*$ & $*$ \\
\hline Chorrúo (2) & $\mathrm{Sp}$ & $\mathrm{A}$ & G & $\mathrm{L}$ & $\mathrm{D}$ & $\mathrm{C}$ & $*$ & $*$ \\
\hline Chorrúo de Fruto Redondo & $\mathrm{Sp}$ & A & $\mathrm{G}$ & $\mathrm{A}$ & $\mathrm{U}$ & $\mathrm{D}$ & $*$ & * \\
\hline Dam & $\mathrm{Sr}$ & A & $\mathrm{D}$ & $\mathrm{B}$ & $\mathrm{U}$ & A & $*$ & $*$ \\
\hline Dolce Agogia & It & A & $\mathrm{J}$ & I & $\mathrm{L}$ & $\mathrm{B}$ & $*$ & $*$ \\
\hline Domat & $\mathrm{Tq}$ & A & $\mathrm{U}$ & $\mathrm{B}$ & $\mathrm{K}$ & $\mathrm{D}$ & $*$ & $*$ \\
\hline Dulzal & $\mathrm{Sp}$ & $\mathrm{A}$ & - & $\mathrm{B}$ & $\mathrm{U}$ & $\mathrm{c}$ & $*$ & $*$ \\
\hline Dulzal-1 & $\mathrm{Sp}$ & $\mathrm{A}$ & $\mathrm{R}$ & E & G & $\mathrm{c}$ & $*$ & $*$ \\
\hline Edremit Yaglik & $\mathrm{Tq}$ & A & $\mathrm{u}$ & $\mathrm{B}$ & $\mathrm{w}$ & $\mathrm{c}$ & $*$ & $*$ \\
\hline Empeltre (2) & $\mathrm{Sp}$ & $\mathrm{A}$ & I & $\mathrm{B}$ & $\mathrm{c}$ & $\mathrm{D}$ & $*$ & $*$ \\
\hline Escarabajuelo-1 & $\mathrm{Sp}$ & $\mathrm{A}$ & $\mathrm{A}$ & $\mathrm{D}$ & $\mathrm{D}$ & $\mathrm{c}$ & $*$ & $*$ \\
\hline Escarabajuelo de Ubeda & $\mathrm{Sp}$ & A & $\mathrm{K}$ & I & $\mathrm{D}$ & $\mathrm{D}$ & $*$ & $*$ \\
\hline Frantoio & It & A & $\mathrm{J}$ & $\mathrm{I}$ & 0 & $\mathrm{~F}$ & $*$ & $*$ \\
\hline Frantoio A. Corsini & It & $\mathrm{A}$ & $\mathrm{J}$ & I & $\mathrm{F}$ & $\mathrm{F}$ & $*$ & $*$ \\
\hline Galego (2) & $\mathrm{Pt}$ & $\mathrm{A}$ & S & $\mathrm{K}$ & G & $\mathrm{C}$ & $*$ & $*$ \\
\hline Genovesa & Sp & $\mathrm{A}$ & $\mathrm{J}$ & $\mathrm{B}$ & $\mathrm{D}$ & $\mathrm{D}$ & c & $\mathrm{c}$ \\
\hline Golbina de Belchite & $\mathrm{Sp}$ & A & $\mathrm{C}$ & $\mathrm{E}$ & $\mathrm{F}$ & $\mathrm{H}$ & * & $*$ \\
\hline Gordal Sevillana (5) & $\mathrm{Sp}$ & A & $\mathrm{L}$ & $\mathrm{F}$ & $\mathrm{D}$ & $\mathrm{c}$ & $\mathrm{d}$ & * \\
\hline Habichuelero & $\mathrm{Sp}$ & A & $\mathrm{B}$ & $\mathrm{D}$ & $\mathrm{D}$ & $\mathrm{D}$ & $*$ & $*$ \\
\hline Hojiblanca (3) & $\mathrm{Sp}$ & A & I & $\mathrm{C}$ & $\mathrm{B}$ & $\mathrm{c}$ & $*$ & $*$ \\
\hline Hrai Souni & $\mathrm{Sr}$ & A & B & $\mathrm{G}$ & $\mathrm{O}$ & $\mathrm{c}$ & e & $*$ \\
\hline
\end{tabular}


Table 1. (continued).

\begin{tabular}{|c|c|c|c|c|c|c|c|c|}
\hline \multirow[b]{2}{*}{ Cultivar } & \multirow[b]{2}{*}{ Origin } & \multicolumn{5}{|c|}{ Banding patterns } & \multicolumn{2}{|c|}{ Identification $^{x}$} \\
\hline & & $\mathrm{ADH}$ & $\mathrm{ME}$ & EST & GPI & LAP & SO & $\mathrm{CO}$ \\
\hline Imperial & $\mathrm{Sp}$ & $\mathrm{A}$ & $\bar{U}$ & $\mathrm{~A}$ & $\mathrm{C}$ & $\mathrm{H}$ & $*$ & $*$ \\
\hline Imperial de Jaén & $\mathrm{Sp}$ & $\mathrm{A}$ & $\mathrm{G}$ & A & $\mathrm{G}$ & $\mathrm{C}$ & $*$ & $*$ \\
\hline Itrana & It & $\mathrm{A}$ & $A$ & $\mathrm{E}$ & $\mathrm{N}$ & $\mathrm{C}$ & $*$ & $*$ \\
\hline Jabaluna & $\mathrm{Sp}$ & $\mathrm{A}$ & $\mathrm{K}$ & $\mathrm{U}$ & $\mathrm{D}$ & $\mathrm{D}$ & $*$ & $*$ \\
\hline Jaropo & $\mathrm{Sp}$ & A & B & $\mathrm{E}$ & $\mathrm{D}$ & $\mathrm{C}$ & $*$ & $*$ \\
\hline Kaesi & $\mathrm{Sr}$ & A & D & $B$ & $P$ & $A$ & $*$ & $*$ \\
\hline Kalamon & $\mathrm{Gr}$ & $\mathrm{B}$ & $\mathbf{N}$ & $\mathrm{L}$ & $\mathrm{R}$ & $\mathrm{C}$ & * & $*$ \\
\hline Kelb et Ter & $\mathrm{Sr}$ & A & - & $\mathrm{F}$ & $\mathrm{R}$ & $\mathrm{H}$ & * & $*$ \\
\hline Kelb et Ter-10/13 & $\mathrm{Sr}$ & $\mathrm{A}$ & B & $\mathrm{C}$ & $\mathrm{O}$ & $\mathrm{C}$ & $*$ & $*$ \\
\hline Koroneiki & $\mathrm{Gr}$ & A & $\mathbf{M}$ & B & W & $\mathrm{H}$ & $*$ & $*$ \\
\hline Leccino & It & A & $\mathbf{T}$ & B & $\mathbf{J}$ & $\mathrm{E}$ & $*$ & $*$ \\
\hline Leccio di Corno & It & A & $\mathrm{Y}$ & B & I & $\overline{\mathrm{E}}$ & $*$ & $*$ \\
\hline Lechín de Granada & $\mathrm{Sp}$ & $A$ & W & $A$ & $\mathrm{~N}$ & $\bar{C}$ & * & $*$ \\
\hline Lechín de Sevilla (6) & $\mathrm{Sp}$ & A & B & G & $\mathrm{O}$ & $\mathrm{C}$ & $\mathrm{e}$ & $*$ \\
\hline Limoncillo (2) & Sp & A & $\mathrm{A}$ & A & $\mathrm{C}$ & $\mathrm{D}$ & $*$ & $*$ \\
\hline Macho de Jaén & $S p$ & A & B & B & $\mathrm{C}$ & $\mathrm{H}$ & * & $*$ \\
\hline Madural & $\mathrm{Pt}$ & $A$ & B & $\mathrm{E}$ & $\mathrm{D}$ & $\mathrm{D}$ & $\mathbf{k}$ & $\mathbf{k}$ \\
\hline Manzanilla de Jaén (3) & Sp & A & B & I & $\mathrm{C}$ & $\mathrm{D}$ & $*$ & $*$ \\
\hline Manzanilla del Piquito & Sp & A & $\mathrm{A}$ & A & $\mathrm{C}$ & $\mathrm{C}$ & f & $\mathbf{f}$ \\
\hline Manzanilla Prieta & $\mathrm{Sp}$ & $\mathrm{A}$ & $\mathrm{G}$ & A & $\mathrm{C}$ & $\mathrm{C}$ & * & $*$ \\
\hline Manzanilla de Sevilla (7) & $\mathrm{Sp}$ & A & $\mathbf{B}$ & $F$ & $\mathrm{G}$ & $\mathrm{C}$ & * & $*$ \\
\hline Manzanilla de Tortosa & $\mathrm{Sp}$ & A & $\mathbf{N}$ & B & $\mathrm{E}$ & $\mathrm{C}$ & * & $*$ \\
\hline Maoui Stambouli & $\mathrm{Sr}$ & A & B & $\mathrm{F}$ & $\mathrm{O}$ & $\mathrm{C}$ & $*$ & $*$ \\
\hline Marteño-10/14 & $\mathrm{Sp}$ & A & G & G & M & $\mathrm{C}$ & $*$ & $*$ \\
\hline Maurino & It & A & $\mathrm{T}$ & I & $\mathrm{F}$ & $\mathrm{E}$ & $*$ & $*$ \\
\hline Megaritiki & $\mathrm{Gr}$ & $\mathrm{B}$ & $\mathbf{J}$ & $\mathbf{T}$ & $\mathrm{V}$ & $\mathrm{H}$ & $*$ & $*$ \\
\hline Memecik & $\mathrm{Tq}$ & A & $\mathrm{C}$ & B & A & A & $*$ & $*$ \\
\hline Merhavia & Is & A & - & $\mathrm{F}$ & $\mathrm{D}$ & $\mathrm{D}$ & $\mathrm{g}$ & $*$ \\
\hline Meski & $\operatorname{Tn}$ & A & $\mathbf{P}$ & $\mathbf{S}$ & $\mathrm{D}$ & $\mathrm{H}$ & $*$ & $*$ \\
\hline Morejona & $\mathrm{Sp}$ & $\mathrm{A}$ & $\mathrm{E}$ & $\mathrm{L}$ & $\mathrm{G}$ & $\mathrm{D}$ & $*$ & $*$ \\
\hline Morona & $\mathrm{Sp}$ & A & $\mathrm{E}$ & $\mathbf{E}$ & $\mathrm{G}$ & $\mathrm{C}$ & $*$ & $*$ \\
\hline Morruda & $S p$ & A & $N$ & B & W & $\mathrm{C}$ & $*$ & $*$ \\
\hline Nabali & Is & A & $\mathrm{B}$ & B & $\mathrm{C}$ & $\mathrm{D}$ & $\mathrm{i}$ & $*$ \\
\hline Negral & $\mathrm{Sp}$ & A & $\mathrm{C}$ & B & $\mathrm{F}$ & $\mathrm{H}$ & $*$ & $*$ \\
\hline Negrillo de Arjona & $\mathrm{Sp}$ & A & - & $\mathrm{E}$ & $\mathrm{B}$ & $\mathrm{D}$ & $*$ & $*$ \\
\hline Negrillo de Estepa & $\mathrm{Sp}$ & A & B & $\mathbf{F}$ & $\mathbf{N}$ & $\mathrm{H}$ & $*$ & $*$ \\
\hline Negrillo de la Carlota & $\mathrm{Sp}$ & A & $\mathbf{G}$ & I & $\mathbf{N}$ & $\mathrm{C}$ & * & $*$ \\
\hline Nevadillo Blanco de Jaen & $\mathrm{Sp}$ & A & $A$ & $\mathrm{C}$ & $\mathbf{M}$ & $\mathrm{C}$ & * & $*$ \\
\hline Nevadillo Blanco de Lucena & $\mathrm{Sp}$ & A & B & $A$ & $\mathrm{C}$ & $\mathrm{C}$ & * & $*$ \\
\hline Nevadillo Blanco de Osuna & $\mathrm{Sp}$ & A & $\mathrm{G}$ & $\mathrm{C}$ & $\mathbf{N}$ & $\mathrm{C}$ & * & $*$ \\
\hline Nevadillo Negro de Jaén & $\mathrm{Sp}$ & A & $\mathrm{Y}$ & $\mathbf{E}$ & $\mathrm{D}$ & $\mathrm{D}$ & $*$ & $*$ \\
\hline Nevadillo-2 & $\mathrm{Sp}$ & A & $\mathrm{O}$ & $\mathbf{L}$ & $\mathrm{D}$ & $\mathrm{D}$ & * & $*$ \\
\hline Nevado Azul (2) & $\mathrm{Sp}$ & A & $\mathrm{B}$ & $\mathbf{B}$ & $\mathrm{C}$ & $\mathrm{C}$ & * & $*$ \\
\hline Nevado Basto & $\mathrm{Sp}$ & A & A & A & $\mathrm{C}$ & $\mathrm{C}$ & f & $f$ \\
\hline Oblonga & U.S & A & $\mathrm{J}$ & G & $Q$ & $\mathrm{~F}$ & $*$ & - \\
\hline Ocal & $\mathrm{Sp}$ & A & $\mathrm{B}$ & $\mathbf{F}$ & $\mathrm{D}$ & D & $\mathrm{g}$ & $*$ \\
\hline Ocal-1 & $\mathrm{Sp}$ & A & I & B & $\mathrm{D}$ & $\mathrm{H}$ & $*$ & $*$ \\
\hline Olivo de Maura & $\mathrm{Sp}$ & A & W & $\mathrm{B}$ & $\mathbf{M}$ & $\mathrm{C}$ & $*$ & $*$ \\
\hline Olivo Macho-3 & $\mathrm{Sp}$ & A & $\mathrm{A}$ & B & $\mathrm{D}$ & D & $\mathrm{b}$ & $\mathrm{b}$ \\
\hline Ouslati & Tn & A & $\mathrm{A}$ & $\mathrm{U}$ & $\mathrm{J}$ & $\mathrm{H}$ & * & $*$ \\
\hline Pajarero de Lucena & $\mathrm{Sp}$ & A & A & $\mathrm{F}$ & $\mathrm{G}$ & $\mathrm{C}$ & $*$ & $*$ \\
\hline Pavo & $S p$ & A & $\mathrm{O}$ & $\mathrm{F}$ & $\mathrm{C}$ & $\mathrm{H}$ & $*$ & $*$ \\
\hline Pendolino & It & A & $\mathrm{E}$ & $\mathrm{B}$ & I & $\mathrm{H}$ & $*$ & $*$ \\
\hline Perillo de Jaén & $S p$ & A & $\mathrm{E}$ & $\mathrm{C}$ & $\mathbf{M}$ & $\mathrm{H}$ & * & $*$ \\
\hline Pico Limón & $\mathrm{Sp}$ & A & 0 & A & $\mathbf{N}$ & $\mathrm{D}$ & $*$ & $*$ \\
\hline Picual (29) & $\mathrm{Sp}$ & A & A & A & $\mathrm{G}$ & $\mathrm{D}$ & $*$ & $*$ \\
\hline Picual de Estepa & $\mathrm{Sp}$ & $\mathrm{A}$ & $\mathrm{B}$ & $\mathrm{C}$ & $\mathrm{D}$ & $\mathrm{D}$ & $*$ & $*$ \\
\hline Picudo (5) & $\mathrm{Sp}$ & A & $\mathrm{E}$ & $\mathrm{P}$ & $\mathrm{C}$ & $\mathrm{C}$ & $*$ & $*$ \\
\hline Picudo Blanco & $S p$ & $\mathrm{~A}$ & $\mathrm{~B}$ & $\mathrm{~L}$ & $\mathbf{M}$ & $\mathrm{C}$ & $*$ & $*$ \\
\hline
\end{tabular}




\begin{tabular}{|c|c|c|c|c|c|c|c|c|}
\hline \multirow[b]{2}{*}{ Cultivar } & \multirow[b]{2}{*}{ Origin } & \multicolumn{5}{|c|}{ Banding patterns } & \multicolumn{2}{|c|}{ Identification $^{\mathrm{x}}$} \\
\hline & & $\mathrm{ADH}$ & $\mathrm{ME}$ & EST & GPI & LAP & $\mathrm{SO}$ & $\mathrm{CO}$ \\
\hline Picudo de Fruto Rojo & $S p$ & A & $\mathrm{E}$ & $\mathrm{D}$ & $\mathrm{C}$ & $\mathrm{C}$ & $\mathbf{h}$ & $*$ \\
\hline Picholine & Fr & A & $\mathrm{A}$ & $\mathrm{B}$ & A & A & * & * \\
\hline Picholine Marrocaine & $\mathrm{Mr}$ & A & B & $\mathrm{D}$ & $\mathrm{C}$ & $\mathrm{D}$ & $\mathbf{a}$ & - \\
\hline Racimal de Jaén & $\mathrm{Sp}$ & $\mathrm{A}$ & $\mathrm{I}$ & $\mathrm{B}$ & $\mathrm{D}$ & $\mathrm{D}$ & * & * \\
\hline Rapasayo & $\mathrm{Sp}$ & $\mathrm{A}$ & $\mathrm{B}$ & $\mathrm{F}$ & $\mathrm{N}$ & $\mathrm{C}$ & $*$ & * \\
\hline Razzola & It & A & J & $\mathbf{G}$ & $\mathrm{F}$ & $\mathrm{F}$ & $*$ & * \\
\hline Real Sevillana & $\mathrm{Sp}$ & $\mathrm{A}$ & G & $\mathrm{L}$ & $\mathbf{M}$ & $\mathrm{C}$ & * & * \\
\hline Redondil & $\mathrm{Pt}$ & $\mathrm{A}$ & $\mathrm{B}$ & B & $\mathrm{G}$ & $\mathrm{C}$ & * & * \\
\hline Redondilla de Logroño & $\mathrm{Sp}$ & A & A & $\mathrm{E}$ & $\mathrm{D}$ & $\mathrm{C}$ & $*$ & $*$ \\
\hline Rosciola & It & $\mathrm{A}$ & A & $\bar{J}$ & W & $\mathbf{H}$ & $*$ & * \\
\hline Royal de Calatayud & $\mathrm{Sp}$ & $\mathrm{A}$ & $\mathbf{U}$ & $\mathrm{H}$ & $\mathbf{M}$ & $\mathrm{C}$ & $*$ & * \\
\hline Salgar Redondo & $S p$ & A & A & $\mathrm{F}$ & $\mathrm{C}$ & $\mathrm{C}$ & $*$ & * \\
\hline San Agostino & It & A & A & I & $\mathrm{U}$ & A & $*$ & * \\
\hline Santa Caterina & It & A & $\mathrm{L}$ & $\mathrm{F}$ & $\mathrm{D}$ & $\mathrm{C}$ & $\mathrm{d}$ & * \\
\hline Sefraoui & $\mathrm{Sr}$ & $\mathrm{A}$ & D & $\mathrm{F}$ & 1 & $A$ & * & * \\
\hline Sevillenca & $\mathrm{Sp}$ & A & $\mathbf{N}$ & $\mathrm{F}$ & $\mathbf{T}$ & $\mathrm{H}$ & $*$ & * \\
\hline Sigoise & $\mathrm{Ar}$ & $\mathrm{A}$ & B & $\mathrm{D}$ & $\mathrm{A}$ & D & * & $\ldots$ \\
\hline Sourani & $\mathrm{Sr}$ & A & B & $\mathrm{C}$ & $\mathbf{H}$ & $\mathrm{C}$ & j & * \\
\hline Tanche & Fr & A & --- & $\mathrm{E}$ & $\mathbf{K}$ & A & $*$ & * \\
\hline Tempranillo de Calatayud & $\mathrm{Sp}$ & A & $\mathrm{C}$ & B & $\mathbf{J}$ & $\mathrm{H}$ & $*$ & * \\
\hline Tempranillo de Lucena & Sp & A & I & $\mathrm{C}$ & $\mathrm{G}$ & $\mathrm{C}$ & $*$ & * \\
\hline Temprano & $\mathrm{Sp}$ & B & $\mathrm{K}$ & $\mathrm{O}$ & $\mathrm{T}$ & $\mathrm{C}$ & $*$ & * \\
\hline Torcio de Huelma & $\mathrm{Sp}$ & A & $\mathbf{R}$ & $\mathrm{O}$ & $\mathrm{U}$ & $\mathrm{C}$ & * & * \\
\hline Trylia & $\mathrm{Tq}$ & A & $\mathbf{N}$ & $\mathrm{B}$ & I & $\mathrm{C}$ & * & * \\
\hline Uovo di Piccione & It & A & $\mathrm{V}$ & $\mathrm{F}$ & $\mathrm{C}$ & $\mathrm{C}$ & $*$ & $*$ \\
\hline Uslu & $\mathrm{Tq}$ & $\mathrm{A}$ & $\mathrm{F}$ & A & A & $\mathrm{D}$ & * & * \\
\hline Valanolia & $\mathrm{Gr}$ & A & $\mathbf{N}$ & $\mathrm{B}$ & $\mathrm{D}$ & $\mathrm{C}$ & $*$ & * \\
\hline Verdale & Fr & A & $S$ & $\mathrm{~F}$ & $\mathrm{C}$ & $\mathrm{C}$ & $*$ & $*$ \\
\hline Verdial de Alcaudete (2) & $\mathrm{Sp}$ & $\mathrm{A}$ & B & B & $\mathrm{C}$ & $\mathrm{D}$ & $\mathrm{i}$ & $*$ \\
\hline Verdial de Badajoz & $\mathrm{Sp}$ & $\mathrm{A}$ & $\mathrm{G}$ & $\mathrm{C}$ & $\mathrm{C}$ & $\mathrm{D}$ & * & $*$ \\
\hline Verdial de Huevar (2) & $\mathrm{Sp}$ & A & A & $\mathrm{D}$ & $\mathrm{C}$ & $\mathrm{C}$ & $*$ & $*$ \\
\hline Verdial de Vélez Málaga & $\mathrm{Sp}$ & A & $\mathrm{Z}$ & A & G & $\mathrm{D}$ & $*$ & * \\
\hline Verdilla de Calatayud & $\mathrm{Sp}$ & A & A & 0 & $\mathbf{F}$ & $\mathrm{D}$ & $*$ & $*$ \\
\hline Villalonga & $\mathrm{Sp}$ & $\mathrm{A}$ & $\ldots$ & $\mathrm{V}$ & --- & $\mathrm{C}$ & $*$ & $*$ \\
\hline Zalmati & Tn & A & $\mathbf{U}$ & $\mathbf{Q}$ & $\mathrm{S}$ & $\mathbf{E}$ & $*$ & * \\
\hline Zarza & $\mathrm{Sp}$ & A & B & $\mathrm{C}$ & $\mathbf{H}$ & $\mathrm{C}$ & j & * \\
\hline Zorzariega & Sp & A & $\mathrm{G}$ & I & $A$ & $\mathrm{C}$ & $*$ & $*$ \\
\hline Total identitied cultivars & & & & & & & 132 & 144 \\
\hline Total unidentified cultivars & & & & & & & 23 & 8 \\
\hline
\end{tabular}

Cultivar: when there is more than one tree Per cultivar it is indicated between parentheses.

'Origin: $\mathrm{Ar}=$ Argelia; $\mathrm{Sp}=\mathrm{Spain} ; \mathrm{Fr}=$ France; $\mathrm{Gr}=\mathrm{Greece} ; \mathrm{Is}=$ Israel; It = Italy; Lb = Lebanon; $\mathrm{Mr}=\mathrm{Morocco} \mathrm{Pt}=\mathrm{Portugal} ; \mathrm{Sr}=\mathrm{Syria}$; $\mathrm{Tn}=\mathrm{Tunisia}$; $\mathrm{Tq}=$ Turkey; U.S. = United States.

"Identification: the same letters indicate identical combinations of banding patterns; SO = identification without considering the country of origin; CO $=$ identification considering the country of origin; ${ }^{*}=$ cultivars identified, unique combinations of banding patterns; a-k = cultivars not identified.

bands for enzymatic systems was illustrated with different strips: black (full-colored), white (medium-colored), and dotted (lowcolored), which indicate high, medium, and low activities, respectively. The banding patterns obtained for each enzymatic system are described below.

$A D H$. Only one zone of activity with either one-banded (A) or a two-banded pattern (B) was observed for ADH (Fig. 1). The twobanded pattern was observed only in 'Caninese', 'Kalamon', 'Megaritiki', and 'Temprano'. The rest of the cultivars exhibited the one-banded pattern. These patterns would suggest that ADH is controlled by two alleles at one locus. This needs confirmation by progeny analysis.

$M E$. Twenty-five diffent banding patterns composed of four to eleven bands were recorded for this enzymatic system (Fig. 2). Unique banding patterns were observed for 'Uslu'(F'), 'Beladi' (H), 'Koroneiki' (M), 'Me\&i'(P), 'Buidiego'(Q), and 'Verdial de Vélez Málaga' (Z).

EST. The zymograms of EST consisted of two band groups (EST1 and EST-2) (Fig. 3). The most-anodal EST- 1 group included five bands and the least-anodal EST-2 group included three bands. Variability was observed for both zones. Six out of the 22 patterns obtained were unique: $\mathrm{J}$ for 'Rosciola', $\mathrm{M}$ for 'Arbequina', $\mathrm{N}$ for 'Cellina', Q for 'Zalmati', R for 'Buidiego', and V for 'Villalonga'.

GPI. The zymograms showed two areas of activity. The moreanodal zone (GPI-1) showed variability but was not consistently storable and it was not included in this study. Considerable polymorphism was observed in the GPI-2 zone, where the bands were consistent and repeatable. Twenty-three different banding patterns were observed among the cultivars tested (Fig. 4). Pat- 

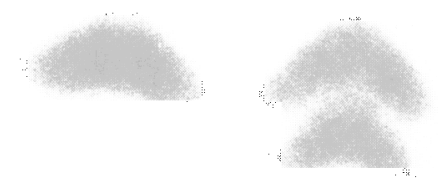

A

B

A
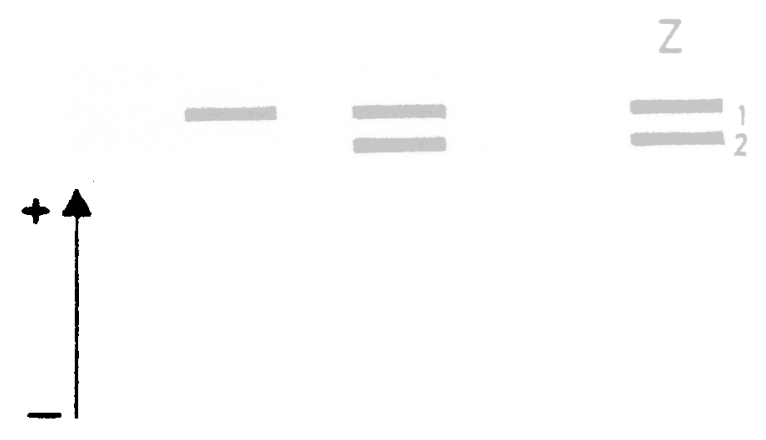

\section{A}

\section{B}

Fig. 1. Photograph and schematic illustration of bands (Z) and banding patterns for ADH.

terns L, P, and S were unique to 'Dolce Agogia', 'Kaesi', and 'Zalmati', respectively.

LAP. Two zones of activity, LAP-1 and LAP-2, were found for this isozyme system. Only the more-anodal (LAP-1) zone showed variability displaying either a single or double-banded pattern (Fig. 5). The LAP-2 zone consisted of two bands present in all cultivars studied. The pattern I was observed only in 'Ascolana Tenera'.

This enzyme is known to be monomeric in other crops (Byrne and Littleton, 1988; Degani and Et-Batsri, 1990; Gottlieb, 1981; Hauagge et al., 1987). LAP-1, based on the banding patterns observed, appears to be controlled by 4 alleles (1-4) at one locus. Under this hypothesis, the patterns B, D, G, and $\mathrm{H}$ would correspond to homozygous genotypes for alleles $3,1,4$, and 2, respectively. Patterns A, C, E, and F would be heterozygous for alleles $1 /$ $3 ; 1 / 2 ; 2 / 4$, and $3 / 4$, respectively. The heterozygote for alleles $2 / 3$ was not observed among the cultivars examined.

Isozyme variability among cultivars. A high level of polymorphism for isozyme phenotypes was observed among the cultivars studied (Table 1). ME, GPI, EST, and LAP were very useful enzymatic systems for varietal identification due to the high number of different banding patterns they yielded. Combinations of these banding patterns allowed the identification of 132 out of 155 cultivars. When the country of origin was considered, 144 out of 155 cultivars could be individually identified. Thus, the method was effective to identify cultivars irrespective of their origin. Therefore, the method should be of great value to identify cultivars in olive germplasm collections.

The relevant enzymatic polymorphism observed among the 155 cultivars tested agrees with the high heterozygosity observed in the species by consequence of their allogamy and with the introgression of genes from wild olives into cultivated forms (Zohary and Spiegel Roy, 1975).

Isoenzymatic variability in olive is comparable to other predominantly outcrossing species like grape (Parfitt and Arulsekar, 1989; Stavrakakis and Loukas, 1983), almond (Cerezo et al., 1989; Hauagge et al., 1987), apple (Weeden and Lamb, 1985), or pineapple (Dewald et al., 1988).

The zymograms obtained for EST, ME, and LAP in this work
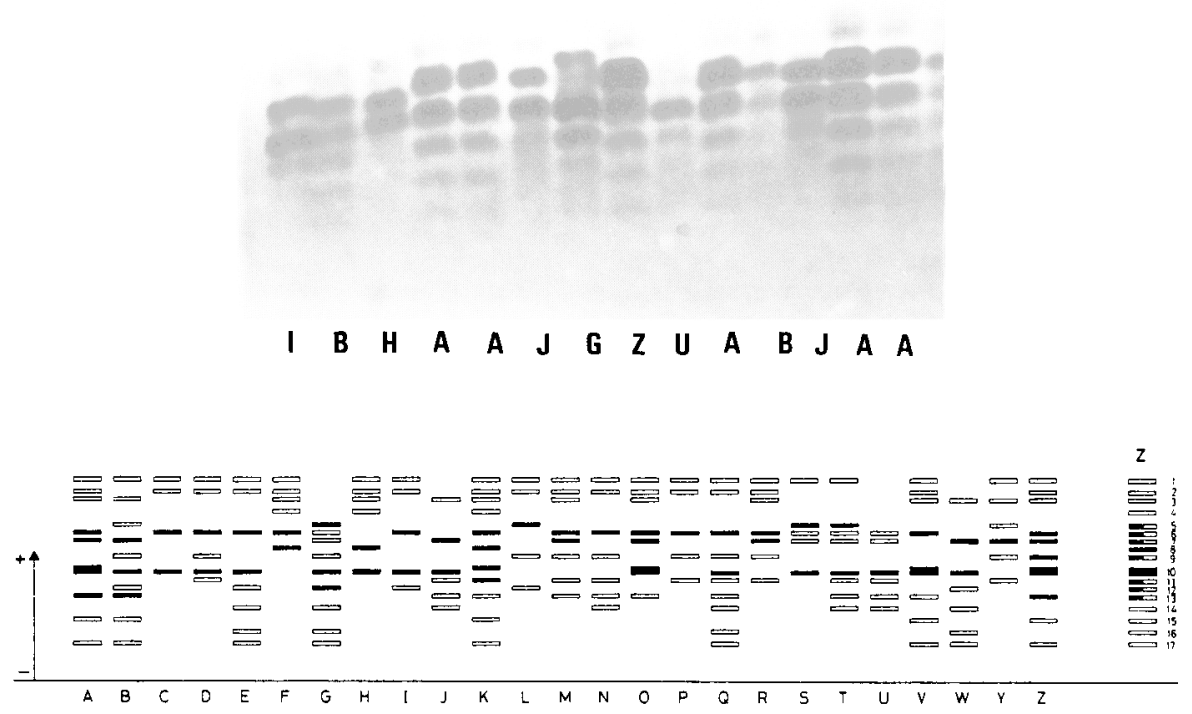

Fig. 2. Photograph and schematic illustration of bands $(\mathrm{Z})$ and banding patterns for ME. 


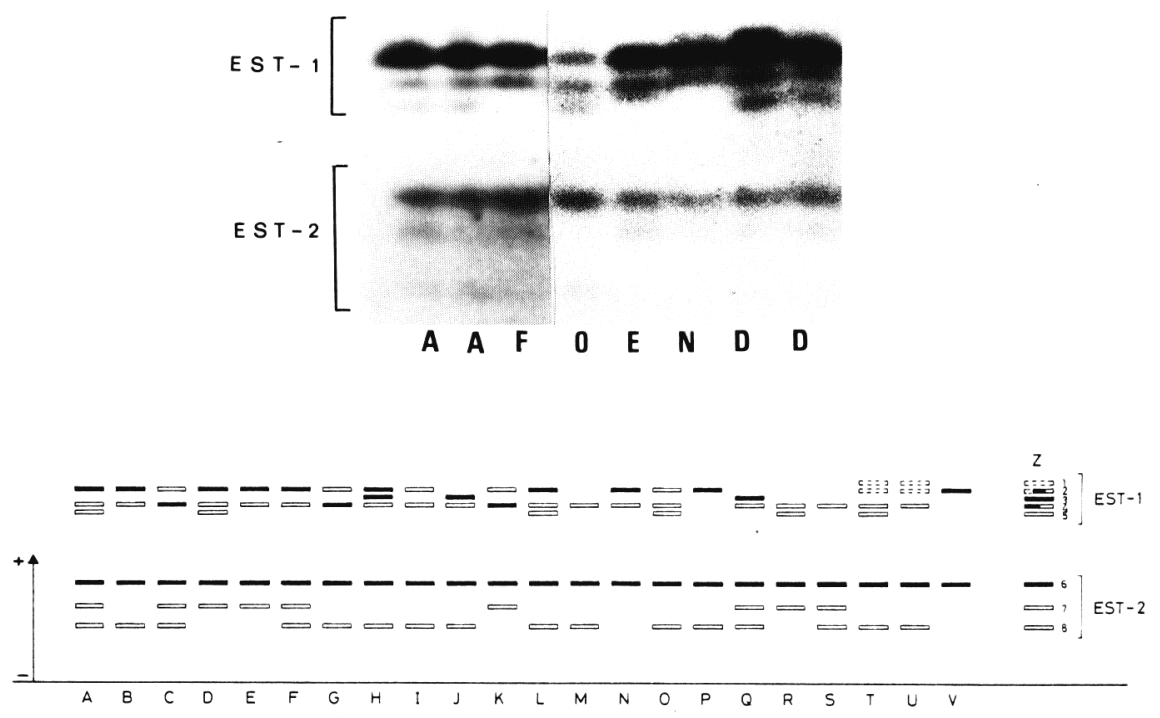

Fig. 3. Photograph and schematic illustration of bands (Z) and banding patterns for EST.

and those previously obtained in olive by Pontikis et al. (1980) are not directly comparable due to the different methodologies used (extraction, gel-electrode buffers, and staining). However both studies report a high level of isozyme polymorphism.

The electrophoretically identical cultivars for all the systems examined could be grouped into ten groups of two cultivars each ('Cañivano Blanco' - 'Picholine Marrocaine'; 'Cañivano Negro'-
'Olivo Macho'; 'Changlot Real' - 'Genovesa'; 'Gordal Sevillana''Santa Caterina'; 'Hrai souni' - 'Lechín de Sevilla'; 'Madural''Cordovil de Serpa'; 'Merhavia' - 'Ocal'; 'Cobrançosa' - 'Picudo de Fruto Rojo'; 'Nabali'-'Verdial de Alcaudete' and 'Sourani''Zarza') and one group of three cultivars ('Ayrouni'-'Manzanilla del Piquito'-'Nevado Basto'). In all cases, when isozyme phenotypes were supplemented with a few precise morphological char-
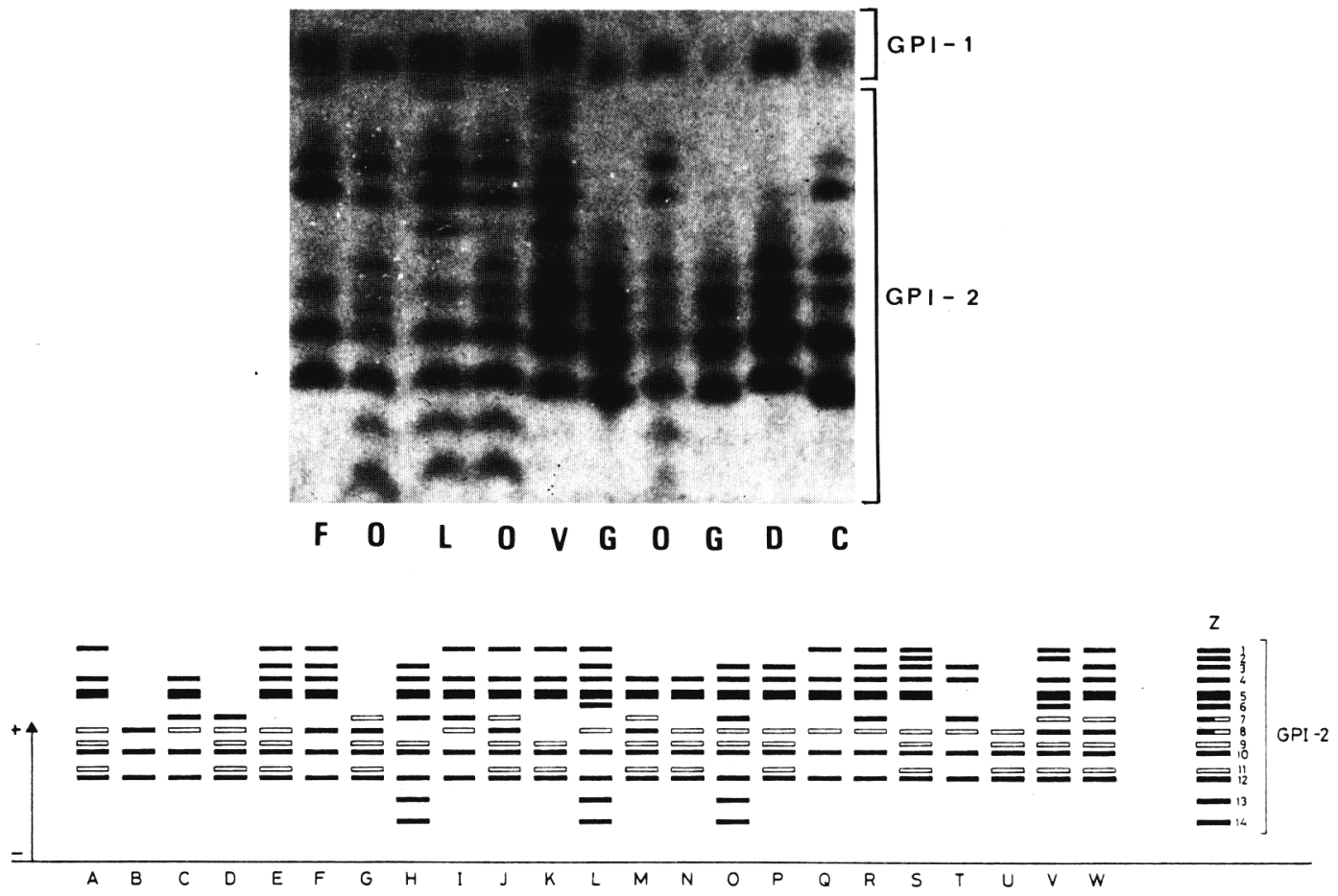

Fig. 4. Photograph and schematic illustration of bands (Z) and banding patterns for GPI. 


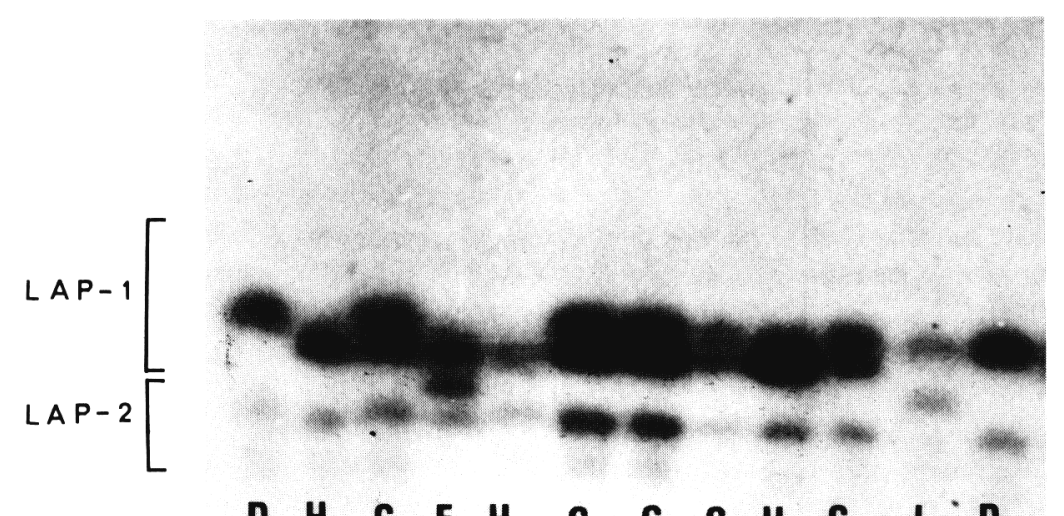

\section{H C E H C C C H C I. D}

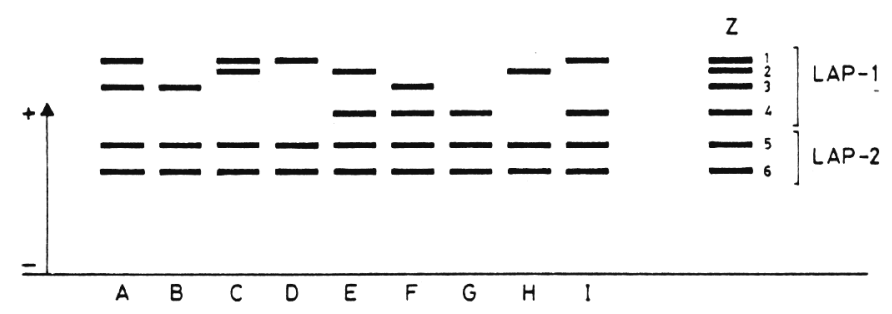

Fig. 5. Photograph and schematic illustration of bands ( $\mathrm{Z}$ ) and banding patterns for LAP.

acteristics of endocarps, leaves, and inflorescence, complete identification of all of the cultivars studied was possible except for 'Madural' and 'Cordovil de Set-pa'. However, our morphological description of these two cultivars disagrees with those obtained by Leitao (1988), suggesting a possible mislabelling in our collection.

Isozyme variability within cultivars. As in other fruit trees, olive cultivars are vegetatively propagated and the only possible intracultivar genetic variation is due to mutation.

Isozymes have been generally unable to detect the genetic changes produced by somatic mutations (DeWald et al, 1988; Tao and Sugiura, 1987; Weeden and Lamb, 1985). However, Loukas and Pontikis (1981) detected slight differences for esterases in two olive samples of the same cultivar from different geographic locations.

We electrophoretically analyzed 90 pollen samples from 21 cultivars collected from the same or different geographic locations. In all cases, no intracultivar polymorphism was observed for the five enzyme systems used. These results provide additional evidence on the lack of intraclone variation for isozyme polymorphisms. Moreover, they strongly support the consistency of the banding patterns obtained with the systems assayed and their . suitability for the identification of olive cultivars.

\section{Literature Cited}

Barranco, D. and L. Rallo. 1984. Las variedades de olive cultivadas en Andalucia. Consejeria de Agricultura de la Junta de Andalucia Ministerio de Agricultura Pesca y Alimentacion, Madrid. Barranco, D. and L. Rallo. 1985. Las variedades de olivo cultivadas en España Olivae 9:16-22.

Bottari, V. and P. Spina. 1953. Le varieta di olivo coltivate in Sicilia. Annali della Sperimentazione Agraria. VII, 937-978; VII, 1141-1174; VII, 1421-1444.

Byrne, D.H. and T.G. Littleton. 1988. Electrophoretic characterization of diploid plums of the Southeastern United States. J. Amer. Soc. Hort. Sci. 113:918-924.

Byrne, D.H. and T.G. Littleton. 1989. Characterization of isozyme variability in apricots. J. Amer. Soc. Hort. Sci. 114:674- 678.

Cerezo, M., R. Socias I Company, and P. Arús. 1989. Identification of almond cultivars by pollen isoenzymes. J. Amer. Soc. Hort. Sci. 114:164-169.
Degani,C. and R. El-Batsri. 1990. Enzyme polymorphism in mango. J. Amer. Soc. Hort. Sci. 115:844-847.

De Wald, M.G. , G.A. Moore, and W.B. Sherman. 1988. Identification of pineapple cultivars by isozyme genotypes. J. Amer. Soc. Hort. Sci. 113:935-938.

Ellstrend, N.C. and J.M. Lee. 1987. Cultivar identification of cherimoya (Annona cherimola Mill) using isozyme markers. Scientia Hort. 32:25-31.

Gottlieb. L.D. 1981. Electrophoretic evidence and plant populations. Prog. Phytochem. 7:1-46. Hauagge, R., D.E. Kester., S. Arulsekar, D.E. Parfitt, and L. Liu. 1987. Isozyme variation among California almond cultivars: Cultivar characterization and origins. J. Amer. Soc. Hort. Sci. 112:693-698.

Leitão, F. 1988. Contribute para o conhecimento de 20 cultivares de 'Olea europaea'L., quer sobre o aspecto de caracterzaçao, quer da productividade, determinante do seu valor economica Dissertaçao. Instituto National de Investigaçao Agraria. Estaçao Agronomica National. Oeiras.

Loukas, M. and C.A. Pontikis. 1981. Is the phenotypic variation observed whithin cultivars of Olea europaea L. reflected in isozyme banding patterns?. J. Hort. Sci. 56(4):377-379.

Loussert, R. and G. Browse. 1980. El olive. Ed. Mundi Prensa, Madrid.

Mora, T.J. and J.M. Serradilla. 1985. Dispersion geografica, diversidad varietal y variabilidad isoenzimática en Vicia faba L. Tesina, Universidad Complutense de Madrid.

Morettini, A. 1972. L'olivicoltura. 2nd ed. Ramo Editoriale degli Agricoltori, Rome.

Pascual, L., F. Perfectti, M. Gutierrez, and A.M. Vargas. 1993. Characterizing isozymes of Spanish cherimoya cultivars. HortScience 28:845-847.

Parfitt, D.E. and S. Arulsekar. 1989. Inheritance and isozyme diversity for GPI and PGM among grape cultivars. J. Amer. Soc. Hort. Sci. 114:486-491.

Pontikis, C.A., M. Loukas, and G. Kousounis. 1980. The use of biochemical markers to distinguish olive cultivars. J. Hort. Sci. 55(4):333-343.

Shields, C.R., T.J. Orton, and C.W. Stuber. 1983. An outline of general resource needs and procedures for the electrophoretic separation of active enzymes from plant tissues, p. 443468. In: S.D. Tanksley and Orton (eds.). Isozymes in plant genetics and breeding. Part A. Elvesier. Amsterdam.

Stavrakakis, M. and M. Loukas. 1983. The between and within grape cultivars genetic variation. Scientia Hort. 36x67-77.

Tao, R. and A. Sugiura. 1987. Cultivars identification of japanese persimmon by leaf isozymes. HortScience 22:932-935.

Valdeyron, G. and P. Crossa-Raynaud. 1950. Les fruits de Tunisie. Annales de Service Botanique et Agronomique de Tunisie 23.

Vallejos, E. 1983. Enzyme activity staining, p. 469-516. In: S.D. Tanksley and T.J. Orton (eds). Isozymes in plant genetics and breeding. Part A. Elsevier, Amsterdam.

Weeden, N.F. and R.C. Lamb. 1985. Identification of apple cultivars by isozyme phenotypes. J. Amer. Soc. Hort. Sci. 110:509-515.

Zohary, A. and P. Spiegel Roy. 1975. Beginnings of fruits growing in the old world. Science 187:319-327. 